$\underline{\text { Preprint typeset in JHEP style. - HYPER VERSION }}$

CERN-TH/99-354

Imperial/TP/99-0/13

\title{
S-Duality and Brane Descent Relations
}

\author{
Laurent Houart \\ Theoretical Physics Group \\ Blackett Laboratory, Imperial College \\ London $S W^{\text {r }}$ 2BZ, UK \\ I.houart@ic.ac.uk \\ Yolanda Lozano \\ Theory Division, CERN \\ 1211 Genève 23, Switzerland \\ yolanda.lozano@cern.ch
}

\begin{abstract}
We present a description of the type IIB NS-NS p-branes in terms of topological solitons in systems of spacetime-filling brane-antibrane pairs. S-duality implies that these spacetime-filling branes are NS9-branes, S-dual to the D9-branes of the type IIB theory. The possible vortex-like solutions in an NS9, anti-NS9 configuration are identified by looking at its worldvolume effective action. Finally we discuss the implications of these constructions in the description of BPS and non-BPS states in the strongly coupled Heterotic $\mathrm{SO}(32)$ theory.
\end{abstract}

KeYwords: D-branes, String Duality. 


\section{Contents}

1. Introduction 1

2. Type IIB Dp-branes as bound states 2

3. The S-dual picture 4

4. Heterotic $\mathrm{SO}(32)$ branes as bound states 6

4.1 The Type I case 6

4.2 The Heterotic case 7

5. Conclusions 10

\section{Introduction}

Recently, very significant progress has been made in our understanding of the role of non-BPS configurations in String Theory, and interesting connections between some BPS branes and non-BPS branes have been uncovered (see [1] for reviews and references therein). It is by now well-known that the BPS Dp-branes of the type II theories can be understood as bound states of a certain number of spacetime-filling branes or brane-antibrane pairs in the theory. In the type IIB case one considers spacetime-filling brane-antibrane pairs built out of BPS type IIB D9-branes charged with respect to the RR 10-form potential [2], whereas in type IIA the spacetime-filling branes are the non-BPS D9-branes introduced by Hor̆ava in [3]. The BPS Dp-branes appear as topological defects in the worldvolume of these unstable systems. An improved understanding of the physics of tachyonic excitations present in unstable branes or brane-antibrane pairs has been the key ingredient for these developments [由]. The presence of tachyons is no longer viewed as a severe pathology of the theory, and a tachyon can be considered like a true Higgs field which tends to develop a stable vacuum expectation value. The simplest example is the description of a BPS Dp-brane in terms of the condensation of the tachyonic mode of the open string stretching between a pair of $\mathrm{D}(\mathrm{p}+2)$ anti-D $(\mathrm{p}+2)$ branes [5], process which can be iterated to construct lower dimensional D-branes by brane descent relations.

Starting with an unstable system of spacetime-filling brane-antibrane pairs, one can analyse the homotopy groups of the vacuum manifold of the Higgs field (tachyon) which appears in the system, and deduce the possible stable defects corresponding 
to stable D-branes [2, 3, 6]. In the type IIB case, the system of coincident D-branes and anti D-branes is in fact characterised by equivalence classes of pairs of vector bundles (one for the D-branes and one for the anti D-branes) which correspond to configurations of spacetime-filling branes up to creation and annihilation, and permit to classify the stable $\mathrm{D} p$-brane charges ${ }^{1}$. These classes appear to be described in a mathematical framework in terms of K-theory groups [7, 2]. More precisely, in the type IIB case the stable $\mathrm{D} p$-brane charges are classified by the K-theory groups $\tilde{K}\left(S^{9-p}\right)$, where the sphere $S^{9-p}$ represents the dimensions transverse to the worldvolume of the $p$-brane (compactified by adding a point at infinity) and $\tilde{K}$ stands for the subgroup of $K$ defined by restricting the equivalence classes to the same number of branes and antibranes. There is a close relation between K-theory (which characterises the conserved D-brane charges and configurations of spacetime-filling branes up to creation and annihilation) and homotopy theory (which classifies the topological defects). The K-theory groups of spheres are equal to the homotopy groups of the vacuum manifold of the Higgs field that appears in the worldvolume theory of the system of spacetime-filling D9 anti-D9 branes.

These beautiful developments in our understanding of BPS and non-BPS states refer mainly to D-branes, and very little is known about possible extensions to the NSNS charged p-branes (see however [8, 9] for some qualitative results). In this paper, we propose to make a step further in this direction and study in the framework of type IIB theory such possibilities. We consider the S-dual picture of the usual spacetime-filling brane-antibrane construction of type IIB, and by studying a system of coincident NS9 anti-NS9 branes discuss the different possible topological defects in the type IIB and Heterotic $\mathrm{SO}(32)$ theories.

The paper is organised as follows. In section 2 we review the construction of the BPS Dp-branes of type IIB as bound states of spacetime-filling D9 anti-D9 pairs. Section 3 discusses the S-dual picture and shows how the NS-NS branes of the type IIB theory can be understood as bound states of NS9 anti-NS9 systems. We then turn in section 4 to the study of the p-branes of the Heterotic $\mathrm{SO}(32)$ theory as bound states of NS9 anti-NS9 branes in a Heterotic background. In particular, we consider the description of the Heterotic SO(32) theory as an orientifold of strongly coupled type IIB by the S-dual of worldsheet parity reversal [10, 11. Finally, section 5 contains the conclusions.

\section{Type IIB Dp-branes as bound states}

In this section we briefly review the construction of the BPS Dp-branes of the type IIB theory as bound states of a certain number of spacetime-filling D9 anti-D9 pairs

\footnotetext{
${ }^{1}$ In type IIA, since one starts with unstable uncharged D9-branes, describing the conserved charges in terms of equivalence classes is less direct [3].
} 
[2]. The BPS Dp-branes appear as topological solitons in the worldvolume of this system, and the explicit way in which they couple can be simply read from the worldvolume effective action of the $9, \overline{9}$ pairs.

The Wess-Zumino part of the worldvolume effective action of a Dp-brane is given by [12]:

$$
S=\int_{\Sigma_{p+1}} C \wedge \operatorname{Tr} e^{F}
$$

where $\mathrm{C}$ is the complex of differential forms which is the sum of the RR potentials. More specifically, in the case of $\mathrm{N}$ coincident D9-branes we are interested in, one has:

$$
\begin{aligned}
S_{\mathrm{D} 9}^{\mathrm{WZ}}= & \int_{R^{9+1}} \operatorname{Tr}\left[C^{(10)}+C^{(8)} \wedge F+C^{(6)} \wedge F \wedge F+C^{(4)} \wedge F \wedge F \wedge F+\right. \\
& \left.+C^{(2)} \wedge F \wedge F \wedge F \wedge F+C^{(0)} F \wedge F \wedge F \wedge F \wedge F\right],
\end{aligned}
$$

where $C^{(p)}$ denotes a $p$-form RR-potential, $F=d b^{(1)} \in \mathrm{U}(\mathrm{N}){ }^{2}$ represents the flux of a fundamental string ending on the branes, and the trace is taken over the $\mathrm{U}(\mathrm{N})$ indices.

The effective action describing brane-antibrane pairs contains additional worldvolume fields. One has, in addition to $F$, a second field strength $F^{\prime}$, associated with the antibranes, and a complex charged tachyon field $T$. The WZ term has a similar but slightly more involved structure [13]. It contains a term :

$$
\int C \wedge\left(e^{F}-e^{F^{\prime}}\right)
$$

along with tachyon-dependent terms which we will not consider here since they will not be important for our discussion. In fact, to discuss the possible realisations of D-branes as topological defects one can focalise on the WZ terms of the brane action (2.2) instead of the ones of the brane-antibrane pairs (2.3), bearing in mind that the topologically non-trivial character of the soliton can be carried by one of the two field strengths, say $F$ (see for instance [5]).

We now turn to the analysis of the WZ terms of (2.2). The second term in this action provides a description of the D7-brane as a bound state D9, anti-D9. The D9, anti-D9 system is unstable due to the existence of a tachyonic mode in the open strings connecting the two types of branes. The tachyon can condense in a topologically non-trivial way [5], and this condensation is accompanied by a localised magnetic flux such that:

$$
\int_{R^{2}} \operatorname{Tr} F=\text { integer . }
$$

\footnotetext{
${ }^{2}$ We do not include explicitly the coupling to the NS-NS 2-form in the field strength of the vector field since it will be irrelevant in the discussion that follows.
} 
Therefore a vortex soliton behaving as a D7-brane is induced, since the integration of $\operatorname{Tr} F$ gives a coupling $\int_{R^{7+1}} C^{(8)}$ in the D9, anti-D9 effective action.

Similarly, the other terms in this action describe other type IIB D $p$-branes as bound states of a certain number of pairs D9, anti-D9. Generically, a $p$-brane can be realised, by a stepwise reasoning, as a bound state of a system of $n(p+2 k)$-brane antibrane pairs for $n=2^{k-1}$ [没], since the homotopy group of the $\mathrm{U}(n)$ vacuum manifold of the $n$ brane-antibrane pairs ${ }^{3}$, which classifies the existence of topologically nontrivial gauge fields on $R^{2 k}$, satisfies: $\Pi_{2 k-1}(\mathrm{U}(n))=Z$. Let us consider for instance the term:

$$
\int_{R^{9+1}} \operatorname{Tr}\left[C^{(6)} \wedge F \wedge F\right]
$$

in the D9, anti-D9 system effective action. $\int_{R^{4}} \operatorname{Tr} F \wedge F$ gives an integer for $F \in U(2)$, since for $k=2 n=2$. The structure of the WZ term implies that this instanton-like configuration carries $C^{(6)}$ charge, and can therefore be identified as a D5-brane. In this way the D5-brane is realised as a bound state of 2 D9, anti-D9 pairs of branes. The structure of the rest of the terms in the WZ action completes a general pattern: D7 $=(\mathrm{D} 9$, anti-D9), D5=2 (D9, anti-D9), D3=4 (D9, anti-D9), D1=8 (D9, anti-D9) and $\mathrm{D}(-1)=16$ (D9, anti-D9), of type IIB D $p$-branes as bound states of D9, anti-D9 pairs. This realisation of the BPS D $p$-branes as topological defects is coherent with the fact that the reduced K-theory groups classifing the conserved D-brane charges in type IIB are given by [2] $\tilde{K}\left(S^{n}\right)$, with $n=9-p$, and that these groups are related to the homotopy groups (see for instance 6]) as: $\tilde{K}\left(S^{n}\right)=\pi_{n-1}(U(N)$ ) for sufficiently large $N$ (i.e. in the stable range). One thus has $\tilde{K}\left(S^{n}\right)=Z$ for $p$ odd and $\tilde{K}\left(S^{n}\right)=0$ for $p$ even.

\section{The S-dual picture}

One important property of the type IIB theory is however missing from the previous construction, namely its S-duality symmetry, also manifest at the level of the $p$-brane solutions. This symmetry implies that if, in particular, the D1- and the D5-branes can be understood as vortex-like solutions in pairs of D9, anti-D9 branes, the same should also be possible for the F1- and NS5-branes, in this case as topological solitons in systems of NS9, anti-NS9 branes.

The so-called NS9-brane is by definition the S-dual of the D9-brane of the type IIB theory, and it is charged with respect to a non-dynamical 10-form NS-NS field, Sdual to the $C^{(10)}$ RR-potential to which the D9-brane is minimally coupled [14. This brane is indeed predicted by the analysis of the type IIB spacetime supersymmetry

\footnotetext{
${ }^{3}$ The gauge group of the $n$ brane-antibrane pairs is $\mathrm{U}(n) \times \mathrm{U}(n)$, but it is broken in the vacuum to the diagonal $\mathrm{U}(n)$ by the tachyon field, and the vacuum manifold of the tachyon is $\mathrm{U}(n) \times \mathrm{U}(n) / \mathrm{U}(n)$, which is topologically equivalent to $\mathrm{U}(n)$.
} 
algebra [15]. Its effective action was constructed in [11] by performing an S-duality transformation in the D9-brane worldvolume effective action. In this reference, and previously in [10], it was also argued that the NS9-brane should play a role in the construction of the Heterotic string with gauge group $\mathrm{SO}(32)$ as a nonperturbative orientifold of the type IIB theory. We will comment on the details of this construction in the next section.

The NS9-brane effective action predicts the F1- and the NS5-branes as bound states of 8 and 2, respectively, NS9 anti-NS9 pairs of branes. The analysis is the same than the one we have just made from the D9-brane worldvolume effective action. The D3-brane, being a singlet under the SL(2,Z) S-duality group, can be realised as a bound state of either D9, anti-D9 or NS9, anti-NS9 branes, since both types of branes carry RR 4-form charge. The same thing happens with the 7-brane and the instanton, though in this case the explicit form of the solutions and worldvolume effective actions describing the branes at strong coupling is different from that in the weak coupling regime (see [16]). In all these configurations the object whose tachyonic mode condenses is a D1-brane, stretched between the NS9, anti-NS9 pairs. The explicit form of the NS9-brane worldvolume effective action reads [11]:

$$
\begin{aligned}
& S_{\mathrm{NS} 9}^{\mathrm{WZ}}=\int_{R^{9+1}} \operatorname{Tr}\left[B^{(10)}+\tilde{C}^{(8)} \wedge \tilde{F}+B^{(6)} \wedge \tilde{F} \wedge \tilde{F}+C^{(4)} \wedge \tilde{F} \wedge \tilde{F} \wedge \tilde{F}+\right. \\
& \left.+B^{(2)} \wedge \tilde{F} \wedge \tilde{F} \wedge \tilde{F} \wedge \tilde{F}+\frac{C^{(0)}}{\left(C^{(0)}\right)^{2}+e^{-2 \phi}} \tilde{F} \wedge \tilde{F} \wedge \tilde{F} \wedge \tilde{F} \wedge \tilde{F}\right] .
\end{aligned}
$$

Here $\tilde{F}$ describes the flux of a D1-brane ending on the NS9-branes ${ }^{4}, B^{(10)}$ is the NS-NS 10-form potential with respect to which the NS9-brane is charged and $\tilde{C}^{(8)}$ is the S-dual of the 8-form RR-potential (see [16]). The second term in this action describes a 7-brane, realised as a bound state of one pair of NS9, anti-NS9 branes. This configuration was predicted in [9], as implied by the realisation of the M-theory Kaluza-Klein monopole as a bound state of a pair M9, anti-M9. The third term in (3.1) is the S-dual of the term: $\int_{R^{9+1}} \operatorname{Tr}\left[C^{(6)} \wedge F \wedge F\right]$, that we discussed in the previous section, and describes a NS5-brane as a bound state of two pairs of NS9, anti-NS9 branes. Similarly, the $B^{(2)}$-term describes a fundamental string as a bound state of eight pairs of NS9, anti-NS9 branes.

The structure of the NS9-brane WZ terms implies that the NS-NS branes of the type IIB theory can be understood as bound states of NS9, anti-NS9 pairs of branes. The general pattern that is derived from this analysis is: D7 $=(\mathrm{NS} 9$, antiNS9), NS5=2 (NS9, anti-NS9), D3=4 (NS9, anti-NS9), F1=8 (NS9, anti-NS9) and $\mathrm{D}(-1)=16$ (NS9, anti-NS9), of type IIB p-branes as bound states of NS9, anti-NS9 pairs. Therefore, we see that all the branes predicted by the analysis of the type IIB spacetime supersymmetry algebra, apart from the pp-wave and the Kaluza-Klein

\footnotetext{
${ }^{4}$ We have again omitted the contribution from the RR 2-form potential.
} 
monopole, can be realised as bound states of any of the two types of spacetime-filling branes of the theory. Of course for this to hold the NS9-branes must be considered on an equal footing with the RR 9-branes. The pp-wave and the Kaluza-Klein monopole solutions are only defined in spacetimes with one special, isometric, direction. In the case of the pp-wave this is the direction of propagation of the wave, whereas in the monopole case this is the Taub-NUT fiber of the transverse space. Therefore these branes cannot be understood as bound states of spacetime-filling branes that do not see any of these special directions.

\section{Heterotic $\mathrm{SO}(32)$ branes as bound states}

In this section we discuss how the solitonic branes of the Heterotic $\mathrm{SO}(32)$ theory can be understood as bound states of spacetime-filling branes. We start by reviewing the type I case, since our description will strongly rely on the S-duality connection between the two theories.

\subsection{The Type I case}

All stable branes in the type I theory, including also non-BPS ones, can be realised as bound states of a certain number of D9, anti-D9 pairs of branes [2]. The definition of the type I theory as an orientifold of type IIB by its worldsheet parity reversal symmetry makes clear that the analysis of section 2 holds straightforwardly in this case, with the only consideration of the projections induced by the orientifold construction in the different gauge fields. In particular the $\mathrm{U}(\mathrm{N})$ gauge group of a set of $\mathrm{N}$ coincident D-branes is broken to $\mathrm{SO}(\mathrm{N})$ for D1- and D9-branes and to $\mathrm{Sp}(\mathrm{N})$ for D5-branes [17.

The general pattern of stable ${ }^{5}$ type I branes in terms of bound states of D9, antiD9 pairs has been derived in [2], and it is given by: D8 $=(\mathrm{D} 9$, anti-D9), D7=2 (D9, anti-D9), D5=4 (D9, anti-D9), D1=8 (D9, anti-D9), D0=16 (D9, anti-D9), D(-1)=32 (D9, anti-D9). The D8, D7, D0 and D(-1) branes are non-BPS. In the type I theory some stable ${ }^{6}$, non-BPS, p-branes with $\mathrm{p}$ even can be realised as bound states of BPS $(\mathrm{p}+1)$, anti- $(\mathrm{p}+1)$ branes, because the tachyonic mode that survives in the spectrum in the type IIB case can be projected out by the orientifold construction for certain values of $\mathrm{p}$ [5], [19]. In particular the $\mathrm{D} 8$ can be obtained as $\mathrm{D} 8=(\mathrm{D} 9$, anti-D9) and the $\mathrm{D} 0$ as $\mathrm{D} 0=(\mathrm{D} 1$, anti-D1) $=16$ (D9, anti-D9). The D7 and D-instanton are obtained as: $\mathrm{D} 7=(\mathrm{D} 7$, anti-D7), $\mathrm{D}(-1)=(\mathrm{D}(-1)$, anti- $\mathrm{D}(-1))$ from type IIB branes, since in these cases the orientifold projection exchanges the brane with the antibrane

\footnotetext{
${ }^{5}$ The D7 and D8 non-BPS branes are however unstable due to the presence of a tachyonic mode in the open strings with one end on the brane and the other on one of the 32 D9-branes [18.

${ }^{6}$ The brane can end up however being unstable due to the presence of tachyonic modes in the open strings with one end on the 32 D9-branes. This happens to be the case for the D7 and D8 branes, as we have just mentioned.
} 
and eliminates the tachyon. Therefore, D7=(D7, anti-D7) $=2(\mathrm{D} 9$, anti-D9), D($1)=(\mathrm{D}(-1)$, anti-D $(-1))=32(\mathrm{D} 9$, anti-D9), using that in type IIB: D7=(D9, anti-D9), $\mathrm{D}(-1)=16(\mathrm{D} 9$, anti-D9) [2].

The general pattern of stable type I branes shows in particular that a same BPS brane in the type I and type IIB theories can be obtained as a bound state of a different number of spacetime-filling branes and antibranes. A concrete example of how the number of spacetime-filling branes required in the construction changes from the type IIB to the type I theory is the D5-brane, discussed in [2]. In the previous section we showed how $\Pi_{3}(U(2))=Z$ implied that the type IIB D5-brane could be understood as an instanton-like configuration from 2 pairs of D9, anti-D9 branes. In the type I theory the gauge group of a single D5-brane is $\operatorname{Sp}(1)=\mathrm{SU}(2)$, and, as discussed in [2] (see also the review by Schwarz in ref. [1]), this seems to be why one needs, in the construction, four D9, anti-D9 pairs, characterised by a $\mathrm{SO}(4) \times \mathrm{SO}(4)$ gauge symmetry. Indeed, in the review of Schwarz [1] it is argued that one of the $\mathrm{SU}(2)$ of the $\mathrm{SO}(4)$ Chan-Paton group characterising say the four anti-D9 branes, is used in the construction of the D5-brane as an instanton configuration and the other one survives as its gauge symmetry ${ }^{7}$.

The D1-brane however is constructed from the same number of D9, anti-D9 pairs of branes in the type I and type IIB theories. One may wonder how this is compatible with the intermediate step in which a D1-brane is realised as a bound state (D5, anti-D5) in type I [5], whereas in the type IIB case one has that D1=2 (D5, anti-D5) ${ }^{8}$. The presence of a different number of D5, anti-D5 branes is due to the fact that in the type I theory a single D5-brane carries already an $\mathrm{SU}(2)$ gauge group, and therefore the D1-brane can be realised as a bound state of a single pair D5, anti-D5 (see [5]). Therefore we can conclude that in terms of 9-branes: D1=(D5, anti-D5) $=8$ (D9, anti-D9), using that in type I D5=4 (D9, anti-D9).

\subsection{The Heterotic case}

It was argued in [10] [11] that the Heterotic string with gauge group $\mathrm{SO}(32)$ could be obtained as a nonperturbative orientifold construction of the type IIB theory. This construction is determined by S-duality: If the type I theory can be defined as an orientifold of the type IIB theory by its worldsheet parity reversal symmetry, its Sdual, i.e. the Heterotic $\mathrm{SO}(32)$ should be defined at strong coupling as an orientifold

\footnotetext{
${ }^{7}$ This argument, which seems natural, is however qualitative. Indeed, it is not, a priori, obvious to show explicitely in this construction the connection between the remaining $\mathrm{SU}(2)$ and the gauge group of the D5-brane. We thank the referee for a discussion on this point.

${ }^{8}$ This is implied by the structure of the D5-brane worldvolume effective action, which contains a term:

$$
\int_{R^{5+1}} \operatorname{Tr}\left[C^{(2)} \wedge F \wedge F\right] .
$$

Again an $\mathrm{SU}(2)$ gauge structure is needed in order to have an integer instanton number.
} 
of the type IIB theory by the S-dual operation of worldsheet parity reversal. Perturbatively, this operation coincides with the $(-1)^{F_{L}}$ symmetry of the type IIB theory, where $F_{L}$ is the left-moving fermion number. Modding out the type IIB theory by $(-1)^{F_{L}}$ gives rise to the type IIA string [20], and this is determined by the twisted sector that has to be added in order to restore modular invariance. However one can consider adding anomaly cancelling 9-branes as in the type I theory, and this is the way in which the Heterotic string can be produced. S-duality determines that the background 9-branes must be NS9-branes, and also that, nonperturbatively, $(-1)^{F_{L}}$ can be defined as the operation reversing the orientation of the D-string. This worldsheet operation has associated an orientifold fixed plane with -32 NS-NS charge, and this charge is cancelled through the addition of 32 NS9-branes, with one unit of NS-NS charge. Together they reproduce the gauge sector of the Heterotic $\mathrm{SO}(32)$ supergravity [11], including corrections in $\alpha^{\prime}$ already computed in the literature [21].

The massless spectrum of the Heterotic F-string can be described in terms of open D-strings with both ends on the F1, DD D-strings, and with one end on the F1 and the other on an NS9-brane, DN D-strings, [11]. The DD D-strings contribute with 8 scalars and 8 right-handed Majorana-Weyl spinors, whereas the DN D-strings contribute with 32 left-moving fermions. The analysis is completely analogous to that in [22], which shows that the BPS D-string of the type I theory has the same worldsheet structure than the $\mathrm{SO}(32)$ Heterotic string. In that case open DD and DN F-strings provide the D-string with the right massless field content of the Heterotic. In our case the open F-strings are replaced by open DD and DN D-strings, which have however the same massless sector than the F1-branes. This description of the Heterotic string arises at strong coupling. However, given that the massless states are BPS, it can be as well extrapolated to the weak coupling regime. In this limit the $\mathrm{SO}(32)$ charges tethered to the $\mathrm{F} 1$ are pulled onto its worldsheet, since $\tau_{\mathrm{D} 1} / \tau_{\mathrm{F} 1} \sim 1 / g_{s} \rightarrow \infty$ and the D-strings collapse to a point, giving the usual $\mathrm{SO}(32)$ Heterotic worldsheet currents.

So far the discussion has been focussed on the massless BPS states. The Heterotic $\mathrm{SO}(32)$ theory contains as well perturbative massive states in the spinorial representation of $\mathrm{SO}(32)$, which are non-BPS but stable, given that they are the lightest ones transforming as spinors of $\mathrm{SO}(32)$. Sen [5], [19] showed that the correct way of describing these states at strong coupling is in terms of a weakly coupled type I (D1, anti-D1) system. This configuration is unstable due to the presence of a tachyonic mode in the open strings stretched between the two branes, and moreover, since the two branes are spinors under $\mathrm{SO}(32)^{9}$ the bound state cannot transform as a spinor. However, compactifying the D-strings and switching on a $Z_{2}$ Wilson line the tachyonic mode can condense into a stable configuration different from the vacuum, and transforming as a spinor of $\mathrm{SO}(32)$ [5]. The mass of this configuration

\footnotetext{
${ }^{9}$ A BPS D1-brane carries the quantum numbers of the spinorial representation of $\mathrm{SO}(32)$ [23].
} 
was also computed in [5] and shown to correspond to a D-particle $\sqrt{2}$ times heavier than the BPS D-particle of the type IIA theory. In the convention of the second reference in [1] this mass reads: $M=\frac{\sqrt{2}}{\sqrt{\alpha^{\prime}}} \frac{1}{g_{I}}$. At strong coupling one has, in the Heterotic side:

$$
M=\frac{\sqrt{2}}{\sqrt{\alpha^{\prime}}} \sqrt{g_{H}},
$$

and this allows to determine how the mass of the perturbative spinorial states in the Heterotic theory, given by $M=\frac{2}{\sqrt{\alpha^{\prime}}}$, gets renormalised as one increases the Heterotic coupling constant.

We can now take the following point of view to describe the spinorial non-BPS states of the Heterotic theory in the strong coupling regime. A bound state $\mathrm{D} 0=(\mathrm{D} 1$, anti-D1) in the weakly coupled type I side predicts a bound state (F1, anti-F1) in the strongly coupled Heterotic theory. S-duality determines that each of these F1's transforms in the spinor representation of $\mathrm{SO}(32)$, since DN open D-strings contribute with these quantum numbers, and that the whole system is unstable due to the presence of a tachyonic mode in the open D-strings stretched between the two F1's. S-duality determines as well that if the system is compactified a state transforming as a spinor under $\mathrm{SO}(32)$ should emerge after the condensation of the tachyonic mode. The mass of this state is formally given by the same expression as in the weakly coupled type I case. In the D0 $=(\mathrm{D} 1$, anti-D1) system the mass is evaluated at the critical radius $R_{c}$ where the effective mass square of all the tachyonic excitations becomes non-negative and the two lightest ones have zero mass [5]. This yields to $M_{\mathrm{D} 0}=2\left(2 \pi R_{c}\right) \tau_{\mathrm{D} 1}$ where $R_{c}=\sqrt{\frac{\alpha^{\prime}}{2}}$. Then it is argued [5] that this mass is actually independent of the radius of the compactification, providing us with the mass of the D-particle in the non-compact limit. Now, in the (F1, anti-F1) Heterotic system the corresponding strong coupling radius is given by $\tilde{R}_{c}=\sqrt{\frac{\alpha^{\prime}}{2}} \sqrt{g_{H}}$ and the mass is $M=2\left(2 \pi \tilde{R}_{c}\right) \tau_{\mathrm{F} 1}=\sqrt{\frac{2}{\alpha^{\prime}}} \sqrt{g_{H}}$, where we used $\tau_{\mathrm{F} 1}=\frac{1}{2 \pi \alpha^{\prime}}$. This provides an alternative interpretation of the result (4.2) in terms of an (F1, anti-F1) bound state. This description at strong coupling is also natural from another point of view. In the Heterotic theory the non-BPS states in the spinorial representation of $\mathrm{SO}(32)$ correspond to unwrapped fundamental strings, i.e. to strings not charged with respect to the NS-NS 2-form potential. This charge cancellation is simply achieved by the F1, anti-F1 superposition.

It is also interesting to point out that the Heterotic fundamental string can arise as a bound state of a pair of NS5, anti-NS5 branes, since this is the S-dual configuration of the $\mathrm{D} 1=(\mathrm{D} 5$, anti-D5) bound state in type $\mathrm{I}$. This is implied by S-duality and can be read as well directly from the NS5-brane effective action truncated to a Heterotic background, in particular from the term [24]: $\int_{R^{5+1}} \operatorname{Tr}\left[B^{(2)} \wedge \tilde{F} \wedge \tilde{F}\right]$. In turn, the NS5-brane is obtained as a bound state of four NS9, anti-NS9 pairs of branes. This is S-dual to the D5=4(D9, anti-D9) configuration in type I, and can also 
be derived from the NS9-brane effective action (3.1) truncated to a Heterotic background $^{10}$. As we discussed in the previous section, from the $\mathrm{SO}(4)$ gauge structure of four D9, anti-D9 pairs of branes only an SU(2) subgroup survives after the instanton construction, and it remains as the gauge group of the D5-brane. In a PolchinskiWitten type of analysis this Yang-Mills multiplet with SU(2) gauge symmetry comes from open strings with both ends on the D5-brane, whereas the D5-brane $\mathrm{SO}(32)$ currents arise from DN open strings, i.e. from strings with one end on the 5-brane and the other on a D9-brane [17]. Hull [10] argued that the same massless modes should be present on the Heterotic 5-brane worldvolume, in this case coming from open D-strings with DD and DN boundary conditions, and with NS9-branes as the spacetime-filling branes. Therefore, the NS5, anti-NS5 pair of branes has an $\mathrm{SU}(2) \times$ $\mathrm{SO}(32)$ gauge structure, from which the $\mathrm{SU}(2)$ is used in the instanton construction of the F-string and the $\mathrm{SO}(32)$ group survives as its gauge structure.

Finally, let us mention that S-duality seems to imply that the S-dual of the non-BPS type I D-instanton has the effect of breaking the $\mathrm{O}(32)$ gauge group of the Heterotic at strong coupling ${ }^{11}$ to its $\mathrm{SO}(32)$ gauge symmetry group (see [2]).

\section{Conclusions}

We have presented a description of the NS-NS p-branes of the type IIB theory in terms of bound states of pairs of NS9, anti-NS9 spacetime-filling branes. This description is determined by the S-duality symmetry of the theory and it is also supported by the worldvolume structure of the NS9-brane. From this construction it is clear that the K-theory groups classifying the conserved NS-NS charges in type IIB are again given by $\tilde{K}\left(S^{9-p}\right)$, but now the spacetime-filling branes are NS9-branes.

Orientifolding this, strongly coupled, type IIB theory by the S-dual of worldsheet parity reversal we have provided a description of the Heterotic $\mathrm{SO}(32)$ branes as topological solitons in systems of NS9, anti-NS9 pairs of branes truncated to a Heterotic background. The picture that emerges is S-dual to the stable brane spectrum of type I in terms of bound states of D9, anti-D9 branes, and gives an interesting description of the strongly coupled non-BPS spinorial states of the Heterotic $\mathrm{SO}(32)$ in terms of bound states (F1, anti-F1), as well as a description of the F-string as a bound state (NS5, anti-NS5).

T-duality on the strongly coupled type IIB theory also gives interesting predictions in the type IIA side. We have seen that the type IIB fundamental string can be obtained as a bound state (NS5, anti-NS5) as: F1=(D3, anti-D3) =2 (NS5, anti-NS5), where the tachyonic mode of a D-string condenses. The T-dual picture describes a fundamental string as a bound state of two NS5, anti-NS5 branes, where

\footnotetext{
${ }^{10}$ This implies that the Heterotic F-string can be obtained as the bound state of eight NS9 brane-antibrane pairs [2]. This can also be read from the NS9-brane effective action.

${ }^{11}$ Because of its duality with weakly coupled type I.
} 
first the tachyonic mode of a D2-brane condenses and then the tachyonic mode of a different brane, a D0-brane: $\mathrm{F} 1=(\mathrm{D} 2$, anti-D2 $)=2\left(\mathrm{NS} 5\right.$, anti-NS5) ${ }^{12}$. From the worldvolume effective action of the type IIA NS5-brane this is described by the term: $\int_{R^{5+1}} B^{(2)} \wedge d a^{(2)} \wedge d c^{(0)}$, where $a^{(2)}$ and $c^{(0)}$ denote, respectively, the 2 -form and scalar worldvolume fields of the NS5A-brane (see [25]).

The T-dual picture of our description of type IIB branes from NS9, anti-NS9 systems gives a realisation of type IIA branes as bound states of NS9A-branes, which are T-dual to the NS9-branes of the type IIB theory. This 9-brane is predicted by the analysis of the type IIA spacetime supersymmetry algebra [15, but contains a Killing direction in its worldvolume, something that is determined both from the T-duality transformation and from its eleven dimensional description as an M9-brane (see [11]), so that it is really a domain-wall type of solution and not a spacetime-filling brane. Therefore, it seems that if one wants to have brane descent constructions which preserve ten dimensional Lorentz invariance one is constrained to use the non-BPS but spacetime-filling D9-branes introduced by Hor̆ava in [3].

Finally, it would also be interesting to analyse similar configurations to the ones studied in this paper in the Heterotic $E_{8} \times E_{8}$ theory. T-duality on the strongly coupled Heterotic $\mathrm{SO}(32)$ theory should provide interesting configurations in the strongly coupled Heterotic $E_{8} \times E_{8}$ side, in its description as a nonperturbative orientifold of the type IIA theory [11]. We hope to report progress in this direction in the near future.

\section{Acknowledgements}

L. H. would like to acknowledge the support of the European Commission TMR programme grant ERBFMBICT-98-2872, and the partial support of PPARC from the grant PPA/G/S/1998/00613.

\section{References}

[1] A. Sen, Non-BPS States and Branes in String Theory, hep-th/9904207; A. Lerda and R. Russo, Stable Non-BPS States in String Theory: a Pedagogical Review, hep-th/9905006; J. H. Schwarz, TASI Lectures on Non-BPS D-Brane Systems, hep-th/9908144; O. Bergman and M. Gaberdiel, NonBPS Dirichlet Branes, hep-th/9908126.

[2] E. Witten, D-Branes and K-Theory, J. High Energy Phys. 9812 (1998) 019, hep-th/9810188.

[3] P. Hor̆ava, Type IIA D-Branes, K-Theory and Matrix Theory, Adv. Theor. Math. Phys. 2 (1999) 1373, hep-th/9812135.

\footnotetext{
${ }^{12}$ We can alternatively consider the process: $\mathrm{F} 1=(\mathrm{D} 4$, anti-D4) $=2(\mathrm{NS} 5$, anti-NS5). Now first a D0-brane condenses and then a D2-brane. These intermediate processes have been discussed in [8, 9].
} 
[4] A. Sen, Stable Non-BPS States in String Theory, J. High Energy Phys. 9806 (1998) 00\%, hep-th/980394; Stable Non-BPS Bound States of BPS D-branes, J. High Energy Phys. 9808 (1998) 010, hep-th/9805019; Tachyon Condensation on the Brane Antibrane system, J. High Energy Phys. 9808 (1998) 012, hep-th/9805170.

[5] A. Sen, SO(32) Spinors of Type I and other Solitons on Brane-Antibrane Pair, J. High Energy Phys. 9809 (1998) 023, hep-th/9808141.

[6] K. Olsen and R. Szabo, Constructing D-Branes from K-Theory, hep-th/9907140.

[7] R. Minasian and G. Moore, K-theory and Ramond-Ramond Charges, J. High Energy Phys. 9711 (1997) 002, hep-th/9710230.

[8] P. Yi, Membranes from Five-Branes and Fundamental Strings from Dp Branes, Nucl. Phys. B550 (1999) 214, hep-th/9901159.

[9] L. Houart and Y. Lozano, Type II Branes from Brane-Antibrane in M-theory, hep-th/9910266.

[10] C.M. Hull, The Non-perturbative SO(32) Heterotic String, hep-th/9812210.

[11] E. Bergshoeff, E. Eyras, R. Halbersma, C.M. Hull, Y. Lozano and J.P. van der Schaar, Spacetime-filling Branes and Strings with Sixteen Supercharges, Nucl. Phys. B (to appear), hep-th/9812224.

[12] M. Douglas, Branes within branes, hep-th/9512077.

[13] C. Kennedy and A. Wilkins, Ramond-Ramond Couplings on Brane-Antibrane Systems, Phys. Lett. B464 (1999) 206, hep-th/9905195.

[14] E. Bergshoeff, M. de Roo, B. Janssen and T. Ortín, The Super D9-brane and its Truncations, Nucl. Phys. B550 (1999) 289, hep-th/9901055.

[15] C.M. Hull, Gravitational Duality, Branes and Charges, Nucl. Phys. B509 (1998) 216, hep-th/9705162.

[16] E. Eyras and Y. Lozano, Exotic Branes and Nonperturbative Seven Branes, Nucl. Phys. B (to appear), hep-th/9908094.

[17] E. Witten, Small Instantons in String Theory, Nucl. Phys. B460 (1996) 541, hep-th/9511030.

[18] M. Frau, L. Gallot, A. Lerda and P. Strigazzi, Stable non-BPS D-branes in Type I String Theory, hep-th/9903123.

[19] A. Sen, Type I D-particle and its Interactions, J. High Energy Phys. 9810 (1998) 021, hep-th/9809111.

[20] A. Dabholkar, Lectures on Orientifolds and Duality, hep-th/9804208. 
[21] A.A. Tseytlin, On SO(32) Heterotic-Type I Superstring Duality in Ten Dimensions, Phys. Lett. B367 (1996) 84, hep-th/9510173; ibid., Heterotic-Type I Superstring Duality and Low Energy Effective Actions, Nucl. Phys. B467 (1996) 383, hep-th/9512081.

[22] J. Polchinski and E. Witten, Evidence for Heterotic-Type I String Duality, Nucl. Phys. B460 (1996) 525, hep-th/9510169.

[23] J. Polchinski, String Theory, Cambridge University Press, 1998.

[24] E. Eyras, B. Janssen and Y. Lozano, 5-branes, KK-monopoles and T-duality, Nucl. Phys. B531 (1998) 275, hep-th/9806169.

[25] E. Bergshoeff, Y. Lozano and T. Ortín, Massive Branes, Nucl. Phys. B518 (1998) 363, hep-th/9712115. 